\title{
Impact of the individualization of the first-line chemotherapy for advanced colorectal cancer based on collagen gel droplet-embedded drug sensitivity test
}

\author{
TAKUMI OCHIAI $^{1}$, KAZUHIKO NISHIMURA ${ }^{1}$, TOMOO WATANABE ${ }^{1}$, MASAYUKI KITAJIMA $^{1}$, \\ AKINORI NAKATANI ${ }^{1}$, KIICHI NAGAYASU ${ }^{1}$, SHIGETOSHI NAITO ${ }^{1}$, TSUYOSHI SATO ${ }^{1}$, KENJI KISHINE ${ }^{1}$, \\ YU ABE ${ }^{1}$, CHIHIRO HARA ${ }^{1}$, SUSUMU YAMADA ${ }^{1}$, SATOMI MASHIKO $^{2}$ and ISAO NAGAOKA ${ }^{3}$ \\ Departments of ${ }^{1}$ Surgery and ${ }^{2}$ Pharmacy, Tobu Chiiki Hospital, \\ Tokyo Metropolitan Health and Medical Treatment Corporation, Tokyo 125-8512; ${ }^{3}$ Department of \\ Host Defense and Biochemical Research, Juntendo University School of Medicine, Tokyo 113-8421, Japan
}

Received August 23, 2016; Accepted February 23, 2017

DOI: $10.3892 / 01.2017 .6960$

\begin{abstract}
Leucovorin (FOL) and fluorouracil (5-FU) plus oxaliplatin (1-OHP; FOLFOX) or FOL and 5-FU plus irinotecan (SN-38; FOLFIRI) are widely used as first-line chemotherapy regimens in the treatment of advanced colorectal cancer (CRC). However, second-line chemotherapy must be abandoned in certain cases due to disease progression, adverse effects or high medical cost. Therefore, the most effective regimen should be selected as first-line chemotherapy. We reported that individualization of first-line treatment (FOLFOX/FOLFIRI/Dual/Poor responder) was possible using the collagen gel droplet-embedded culture drug sensitivity test (CD-DST) and that individualized first-line chemotherapy with CD-DST may improve the prognosis of patients with unresectable CRC. The aim of the present prospective cohort study was to evaluate the individualization of first-line chemotherapy using CD-DST, with a focus on prognosis. Between March 2008 and December 2015, tumor specimens were obtained from 120 patients with CRC who had not received preoperative chemotherapy. CD-DST was performed and the growth inhibition rate (IR) was determined by exposure for $24 \mathrm{~h}$ with $5-\mathrm{FU}$ and 1-OHP $(6.0$ and $3.0 \mu \mathrm{g} / \mathrm{ml}$, respectively) and 5-FU and SN-38 (6.0 and $0.2 \mu \mathrm{g} / \mathrm{ml}$, respectively). The cumulative distribution of IR values under each condition was evaluated on the basis that the clinical response
\end{abstract}

Correspondence to: Dr Takumi Ochiai, Department of Surgery, Tobu Chiiki Hospital, Tokyo Metropolitan Health and Medical Treatment Corporation, 5-14-1 Kameari, Katsushika, Tokyo 125-8512, Japan

E-mail: takumi_ochiai@tokyo-hmt.jp

Key words: colorectal cancer, individualized chemotherapy, collagen gel droplet-embedded drug sensitivity test, leucovorin and fluorouracil plus oxaliplatin, leucovorin and fluorouracil plus irinotecan to FOLFOX and FOLFIRI is equivalent ( $\sim 50 \%)$. The prognosis of dual responder was improved compared with that of poor responders, however this difference was identified to be significant. There was no different prognosis between patients treated with an appropriate first-line regimen and patients treated with an inappropriate first-line regimen in dual responders. However, in poor responders, there were significant differences of prognosis between patients treated with an appropriate first-line regimen and patients treated with an inappropriate first-line regimen $(\mathrm{P}=0.036)$. In conclusion, the results from the present study suggest that administration of the recommended first-line regimen using CD-DST for patients with unresectable CRC is important for the improvement of prognosis, particularly in poor responders.

\section{Introduction}

Colorectal cancer (CRC) is one of the most common cancers worldwide (1). Over the last 20 years, and the last decade in particular, the clinical outcome for metastatic CRC patients has improved greatly due to patients undergoing advanced surgical resection of localized metastasis and advanced systemic chemotherapy $(2,3)$. The leucovorin (FOL) and fluorouracil (5-FU) plus oxaliplatin (1-OHP; FOLFOX) or FOL and 5-FU plus irinotecan (SN-38; FOLFIRI) with molecularly-targeted drugs are used as first-line chemotherapy regimens worldwide in the treatment of advanced CRC $(4,5)$. Recently studies have revealed that the median survival time (MST) of advanced CRC with the chemotherapy was $>30$ months with the integration of multiple cytotoxic agents and molecularly-targeted therapies (6-8). It is common knowledge that the treatment period of the first-line chemotherapy is the longest, and that the response rate of the first-line chemotherapy is the highest (9). However, second-line chemotherapy must be abandoned in certain cases due to disease progression and adverse effects. In addition, high medical costs have been reported to be a significant problem (10-14). Therefore, a more effective regimen should be selected as first-line chemotherapy treatment in a clinical setting. 
A previous report demonstrated that individualization of first-line chemotherapy was possible using the collagen gel droplet-embedded culture drug sensitivity test (CD-DST) and individualized first-line chemotherapy using CD-DST may improve the prognosis of patients with unresectable CRC $(15,16)$.

The aim of this prospective cohort study was to evaluate the individualization of first-line chemotherapy using CD-DST, focusing on prognosis.

\section{Materials and methods}

Patients. During the period between March 2008 and December 2015, tumor specimens were obtained from 120 patients with CRC. Lymph node metastasis and/or distant metastasis was reported in these patients. No patient was treated with preoperative chemotherapy or chemoradiotherapy. Written informed consent for measurement of individual chemosensitivity was obtained from all patients. Approval for the present study was obtained from the Tobu Chiiki Hospital Institutional Review Board (No. 02.03.29. \#1).

Methods. The CD-DST was performed using a Human Cancer Primary Culture System Kit; Primastarä (Kurabo Industries, Ltd., Chuo-ku, Osaka, Japan). Tumor tissue was excised from primary surgical specimens and subjected to the CD-DST. The CD-DST allows for the evaluation of drug sensitivity using isolated 3-dimensionally cultured tumor cells in a small collagen gel droplet, and was used to evaluate the sensitivity of the tumors to 5-FU, which was performed according to a previous description by Kobayashi et al $(17,18)$. Each specimen was washed 5 times with $50 \mathrm{ml}$ saline, followed by additional washing 5 times with $50 \mathrm{ml}$ antibiotic fluid containing $1.0 \mathrm{mg} / \mathrm{ml}$ piperacillin and $0.5 \mathrm{mg} / \mathrm{ml}$ kanamycin. The transport bottle contained $1.0 \mathrm{mg} / \mathrm{ml}$ piperacillin, $0.5 \mathrm{mg} / \mathrm{ml} \mathrm{kanamycin} \mathrm{and} 2.5 \mu \mathrm{g} / \mathrm{ml}$ amphotericin B. Tissue $(1 \mathrm{~g})$ was treated for $2 \mathrm{~h}$ at $37^{\circ} \mathrm{C}$ with a cocktail containing $1.0 \%$ dispersion enzyme EZ ${ }^{\mathrm{TM}}$ (Kurabo Industries, Ltd.). Dispersed cell suspensions were inoculated into pre-culture media on collagen-coated flasks (CG-flusk ${ }^{\mathrm{TM}}$, Kurabo Industries, Ltd.) overnight. Viable tumor cells were subsequently recovered by $0.05 \%$ collagenase treatment. Recovered cells were embedded in $30 \mu$ l collagen gel droplets.

The embedded cells were cultivated in culture media containing 5-FU and 1-OHP at 6.0 and $3.0 \mu \mathrm{g} / \mathrm{ml}$ (FOLFOX regimen), or 5-FU and SN-38 at 6.0 and $0.2 \mu \mathrm{g} / \mathrm{ml}$ (FOLFIRI regimen), respectively, for $24 \mathrm{~h}$ at $37^{\circ} \mathrm{C}$. Following the removal of the anticancer agent-containing media, cells were additionally cultured for 7 days in serum-free culture media (PCM- $2^{\mathrm{TM}}$, Kurabo Japan) to prevent the growth of fibroblasts. Viable cells were stained with neutral red solution and counted using the imaging colorimetric quantification method (Primage ${ }^{\mathrm{TM}}$, Kurabo Japan). The surviving cell number ratio between the drug-treated and control group, which received no drug treatment, was calculated. A growth rate $<0.8$ was regarded a successful culture.

The histograms and the cumulative distributions of the growth inhibition rates (IRs) under the two conditions were evaluated based on the evidence that the clinical response rates to FOLFOX and FOLFIRI were $~ 50 \%(9,19-21)$. Therefore, taking the median of the histogram as the cut off value in each regimen, the patients were divided into responder and poor responder.

All patients were divided into 4 cohorts: FOLFOX and FOLFIRI responder (dual responder), FOLFOX responder, FOLFIRI responder and poor responder.

All patients were divided into 3 cohorts: FOLFOX recommended, FOLFIRI recommended and the two regimens recommended.

The patients with the chemotherapy were divided into 2 cohorts: Treated with appropriate first-line regimen and treated with inappropriate first-line regimen.

First-line regimens were selected by the attending physician. Frequencies of chemotherapy and prognosis were prospectively evaluated and compared among the cohorts.

Statistical analysis. Histograms were analyzed with the D'Agostino-Pearson omnibus normality test. Frequencies of chemotherapy were compared between the two cohorts using the t-test. The MST was calculated by the Kaplan-Meier method. The overall survival (OS) curves of the two cohorts were compared by the log-rank test. Data are presented as the means \pm standard deviation (SD) and were analyzed using GraphPad Prism (version 5.04; GraphPad Software, Inc., La Jolla, CA, USA). $\mathrm{P}<0.05$ was considered to indicate a statistically significant difference.

\section{Results}

Patient characteristics are demonstrated in Table I. The median follow-up period was 1124 days. The individual growth IRs under each of the two conditions are presented in Table II. Histograms of the individual growth IRs (\%) under the conditions of the FOLFOX regimen and FOLFIRI regimen are presented in Figs. 1 and 2, respectively. The median, mean, SD and standard error (SE) of the mean with the FOLFOX regimen were 59.6, 59.5, 16.8 and 1.54, respectively. The median, mean, $\mathrm{SD}$ and SE of the mean with the FOLFIRI regimen were 70.0, $67.7,16.8$ and 1.53 , respectively. The histograms passed the normality test $(\alpha=0.05$; FOLFOX regimen, $\mathrm{P}=0.52$; FOLFIRI regimen, $\mathrm{P}=0.07)$.

Four cohorts (dual, FOLFOX, FOLFIRI, and poor responder). The 4 cohorts based on the cumulative distribution of the individual growth IRs between the two conditions is shown in Fig. 3. Individualization of first-line chemotherapy was possible in all 120 patients, with dual responder, FOLFOX responder, FOLFIRI responder and poor responder in 53, 8, 8 , and 51 patients, respectively. Thirty-nine of the patients eventually received the chemotherapy in dual responder $(n=21)$, FOLFOX responder $(n=3)$, FOLFIRI responder $(n=2)$ and poor responder $(\mathrm{n}=13)$. The MST in dual responder and poor responder was 1128 and 810 days, respectively $(\mathrm{P}=0.119$, Fig. 4).

Three cohorts (FOLFOX, FOLFIRI and the two regimens recommended). Individualization of first-line chemotherapy was possible in all 120 patients, with FOLFOX and FOLFIRI showing higher efficacy in 63 and 51 patients, respectively, and 
Table I. Patient characteristics.

\begin{tabular}{lc}
\hline Variables & Value \\
\hline Age, years, mean (range) & $66.1(36-83)$ \\
Gender & \\
Male/female & $79 / 41$ \\
Histological type & \\
Papillary adenocarcinoma & 2 \\
Well differentiated adenocarcinoma & 22 \\
Moderately differentiated adenocarcinoma & 80 \\
Poorly differentiated adenocarcinoma & 6 \\
Mucinous adenocarcinoma & 9 \\
Squamous cell carcinoma & 1 \\
Primary tumor site & \\
Colon/rectum & $76 / 44$ \\
\hline
\end{tabular}

equal efficacy in 6 cases (Fig. 5). Thirty-nine of the patients eventually received the chemotherapy in FOLFOX recommended $(n=22)$, FOLFIRI recommended $(n=15)$, and the two regimens recommended $(\mathrm{n}=2)$.

Two cohorts (appropriate and inappropriate first-line chemotherapy). Thirty-nine patients with unresectable CRC were treated with chemotherapy. Patients treated with appropriate first-line regimen and those treated with inappropriate first-line regimen were 28 and 11, respectively. All patients treated with inappropriate first-line regimen received FOLFOX therapy (Fig. 5). The MST in patients treated with the appropriate first-line regimen and those treated with an inappropriate first-line regimen was 960 and 506 days, respectively ( $\mathrm{P}=0.218$, Fig. 6$)$. In dual responders, the MST in patients treated with the appropriate first-line regimen $(n=17)$ and those treated with the inappropriate first-line regimen $(n=4)$ was 1044 and 1073 days, respectively ( $\mathrm{P}=0.793$, Fig. 7$)$. In the poor responder group, the MST in patients treated with appropriate first-line regimen $(n=8)$ and those treated with inappropriate first-line regimen $(n=5)$ was 810 and 337 days, respectively $(\mathrm{P}=0.036$, Fig. 8). The mean frequency of appropriate and inappropriate regimen in the two cohorts was $22.8 \pm 5.17$ and $11.0 \pm 1.98$ courses, respectively $(\mathrm{P}=0.142$, Fig. 9$)$.

\section{Discussion}

The present study demonstrated three things. Firstly, the prognosis of a dual responder was improved compared with that of poor responders. Secondly, there was no different prognosis between patients treated with the appropriate first-line regimen and patients treated with an inappropriate first-line regimen in dual responders. Thirdly, in poor responders, there were significant differences in the prognosis between patients treated with an appropriate first-line regimen and patients treated with an inappropriate first-line regimen.

The prognosis of dual responders was improved compared with that of a poor responder. However, there no significant difference was identified between the two cohorts. The reason for this may be that the periods of observation of 4 patients
Table II. Growth inhibition rates (\%) of FOLFOX and FOLFIRI.

\begin{tabular}{|c|c|c|}
\hline Patient no. & FOLFOX & FOLFIRI \\
\hline 1 & 80.1 & 82.9 \\
\hline 2 & 71.3 & 79.2 \\
\hline 3 & 81.2 & 83.4 \\
\hline 4 & 60.0 & 68.7 \\
\hline 5 & 29.9 & 66.5 \\
\hline 6 & 69.7 & 89.6 \\
\hline 7 & 58.7 & 63.2 \\
\hline 8 & 73.0 & 85.2 \\
\hline 9 & 63.2 & 75.9 \\
\hline 10 & 77.9 & 85.5 \\
\hline 11 & 76.3 & 85.6 \\
\hline 12 & 53.6 & 62.6 \\
\hline 13 & 41.9 & 60.7 \\
\hline 14 & 81.3 & 80.9 \\
\hline 15 & 42.3 & 70.2 \\
\hline 16 & 84.8 & 86.8 \\
\hline 17 & 75.9 & 83.9 \\
\hline 18 & 59.2 & 76.4 \\
\hline 19 & 69.9 & 85.5 \\
\hline 20 & 57.0 & 49.7 \\
\hline 21 & 79.2 & 83.0 \\
\hline 22 & 86.1 & 89.1 \\
\hline 23 & 67.3 & 74.4 \\
\hline 24 & 81.3 & 85.2 \\
\hline 25 & 60.4 & 71.9 \\
\hline 26 & 93.4 & 98.6 \\
\hline 27 & 62.6 & 84.9 \\
\hline 28 & 58.5 & 54.8 \\
\hline 29 & 81.2 & 84.0 \\
\hline 30 & 66.5 & 73.3 \\
\hline 31 & 81.3 & 78.1 \\
\hline 32 & 59.9 & 74.1 \\
\hline 33 & 53.3 & 65.0 \\
\hline 34 & 49.3 & 48.3 \\
\hline 35 & 44.7 & 49.3 \\
\hline 36 & 68.8 & 72.1 \\
\hline 37 & 59.7 & 69.4 \\
\hline 38 & 50.8 & 59.3 \\
\hline 39 & 51.6 & 56.5 \\
\hline 40 & 57.9 & 70.2 \\
\hline 41 & 58.5 & 63.7 \\
\hline 42 & 62.4 & 72.5 \\
\hline 43 & 15.2 & 21.5 \\
\hline 44 & 82.9 & 84.3 \\
\hline 45 & 66.3 & 68.1 \\
\hline 46 & 46.7 & 57.2 \\
\hline 47 & 71.3 & 70.0 \\
\hline 48 & 32.6 & 43.2 \\
\hline 49 & 56.4 & 59.2 \\
\hline 50 & 63.4 & 64.6 \\
\hline 51 & 59.7 & 55.3 \\
\hline
\end{tabular}


Table II. Continued.

\begin{tabular}{|c|c|c|c|c|c|}
\hline Patient no. & FOLFOX & FOLFIRI & Patient no. & FOLFOX & FOLFIRI \\
\hline 52 & 46.2 & 45.4 & 104 & 60.1 & 79.2 \\
\hline 53 & 68.7 & 77.7 & 105 & 36.0 & 37.1 \\
\hline 54 & 49.2 & 42.7 & 106 & 59.1 & 81.3 \\
\hline 55 & 31.5 & 43.8 & 107 & 60.1 & 69.4 \\
\hline 56 & 35.5 & 41.5 & 108 & 34.5 & 39.9 \\
\hline 57 & 69.5 & 76.6 & 109 & 39.8 & 47.3 \\
\hline 58 & 57.0 & 53.7 & 110 & 39.0 & 44.9 \\
\hline 59 & 53.7 & 55.8 & 111 & 38.3 & 43.3 \\
\hline 60 & 72.4 & 83.4 & 112 & 39.9 & 40.3 \\
\hline 61 & 29.4 & 43.8 & 113 & 54.6 & 66.8 \\
\hline 62 & 37.7 & 65.1 & 114 & 79.0 & 86.8 \\
\hline 63 & 68.4 & 77.2 & 115 & 88.1 & 95.0 \\
\hline 64 & 47.9 & 73.7 & 116 & 58.6 & 52.0 \\
\hline 65 & 58.8 & 69.1 & 117 & 88.5 & 94.1 \\
\hline 66 & 50.5 & 76.5 & 118 & 84.9 & 84.6 \\
\hline 67 & 74.3 & 81.9 & 119 & 83.3 & 83.7 \\
\hline 68 & 36.4 & 46.3 & 120 & 99.7 & 99.9 \\
\hline
\end{tabular}

71

72

73

74

75

76

77

78

79

80

81

82

83

84

85

86

87

88

89

90

91

92

93

94

95

96

97

98

99

100

101

102

103
68.0

64.3

69.9

40.7

61.4

60.0

44.8

73.3

66.6

27.8

47.7

51.5

59.4

30.6

68.6

66.5

75.0

56.3

58.0

31.0

84.5

42.1

51.1

86.8

54.8

52.0

49.4

23.7

45.4

47.9

64.6

64.9

53.9

45.4
Table II. Continued.

FOLFOX, leucovorin and fluorouracil plus oxaliplatin; FOLFIRI, leucovorin and fluorouracil plus irinotecan.

78.2

83.7

58.0

55.3

79.5

60.2

93.9

79.8

25.5

66.2

62.6

69.9

26.6

75.6

81.8

73.5

57.1

69.0

47.3

89.5

26.9

54.2

92.0

56.9

70.1

71.7

53.5

63.0

52.6

63.5

78.5

61.4

59.9

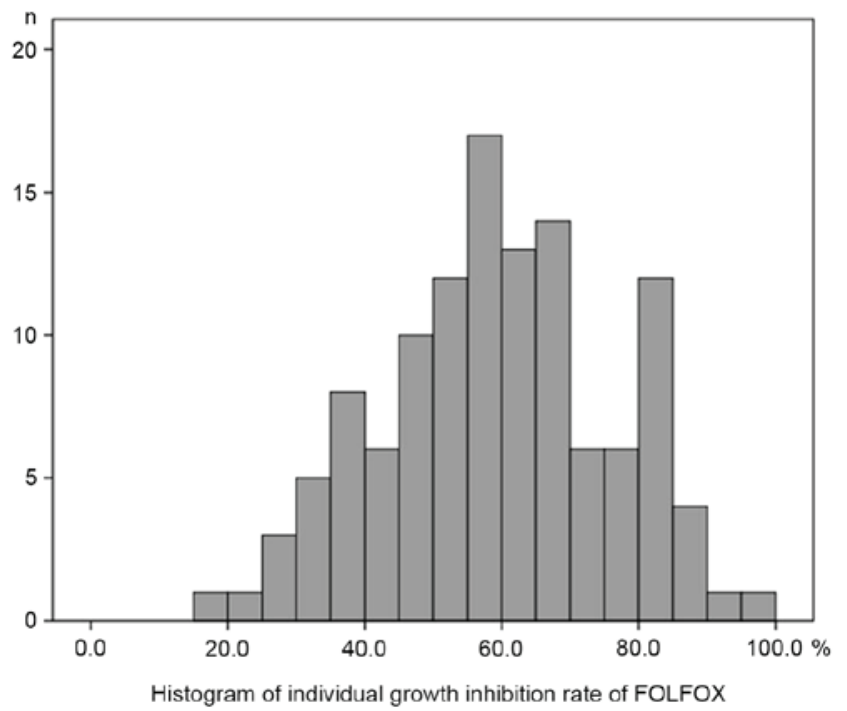

Figure 1. Histogram of individual growth inhibition rate (\%) in culture media containing 5-FU and $1-\mathrm{OHP}$ at 6.0 and $3.0 \mu \mathrm{g} / \mathrm{ml}$ for $24 \mathrm{~h}$ at $37^{\circ} \mathrm{C} .5-\mathrm{FU}$, 5 -fluorouracil; 1-OHP, 1-oxaliplatin; FOLFOX, leucovorin and fluorouracil plus oxaliplatin.

in the dual responder group were $<150$ days. In the dual responder group, the longest-term survivor ( $>2700$ days) was treated with an inappropriate first-line regimen. However, the patient's growth IRs of FOLFOX and FOLFIRI were 77.9 and $85.5 \%$, respectively. These growth IRs were high level. For certain patients, whose growth IRs of FOLFOX and FOLFIRI were high-level, it was not significant whether FOLFOX or FOLFIRI was administered first. This result may support Grothey's report in dual responders. Several studies have 


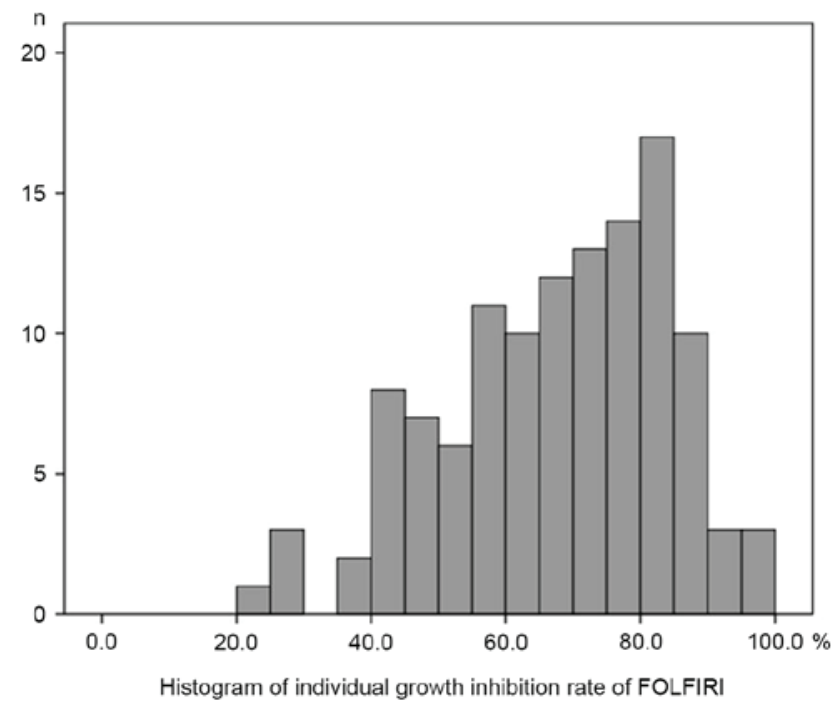

Figure 2. Histogram of individual growth inhibition rate (\%) in culture media containing 5-FU and SN-38 at 6.0 and $0.2 \mu \mathrm{g} / \mathrm{ml}$ for $24 \mathrm{~h}$ at $37^{\circ} \mathrm{C} .5-\mathrm{FU}$, 5 -fluorouracil; SN-38, irinotecan; FOLFIRI, leucovorin and fluorouracil plus irinotecan.

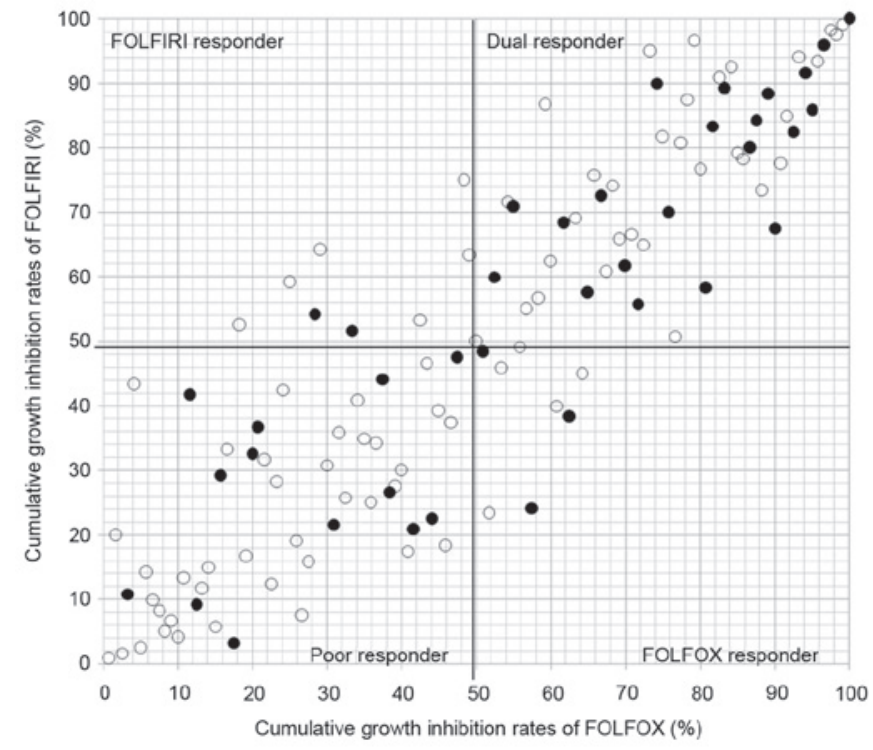

Figure 3. Correlation between cumulative distribution of individual growth inhibition rate between each condition. Solid line indicates cumulative rate $50 \%$. The upper right half area indicates dual responder. The lower right half area indicates FOLFOX responder. The upper left half area indicates FOLFIRI responder. The lower left half area indicates poor responder. Open circle indicates the patients treated without chemotherapy. Closed circle indicates the patients treated with chemotherapy. FOLFOX, leucovorin and fluorouracil plus oxaliplatin; FOLFIRI, leucovorin and fluorouracil plus irinotecan.

investigated individualization in 5-FU-based chemotherapy based on the 5-FU metabolism-associated enzymatic and genetic characteristics of the individual patient (22-29). In addition, the individualization of 5-FU-based chemotherapy based on the serum 5-FU concentration has been reported lately (30-34). However, individualization in 5-FU-based chemotherapy remains to be implemented in a clinical setting. Therefore, CD-DST may be useful to detect poor responder in 5-FU-based chemotherapy. On the other hand,

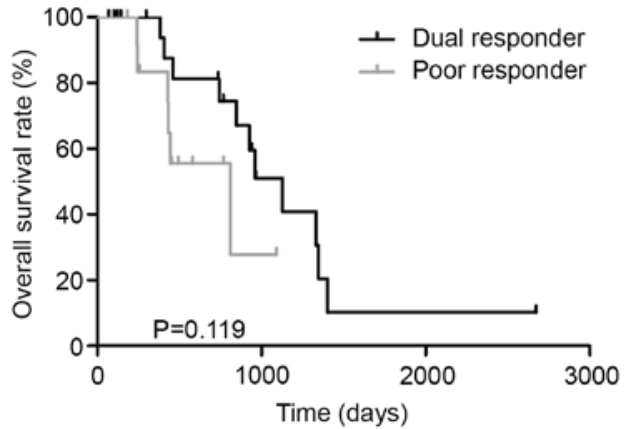

Figure 4. Overall survival rates in dual responder (black line) and poor responder (gray line).

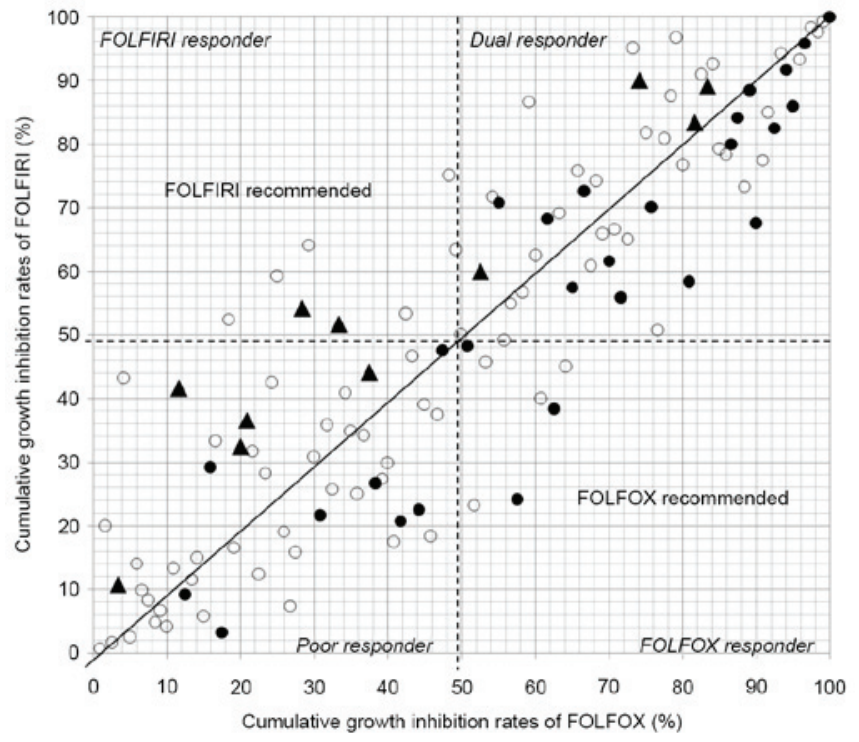

Figure 5. Correlation between cumulative distribution of individual growth inhibition rate between each condition. Solid line indicates equivalence in efficacy for FOLFOX and FOLFIRI. FOLFIRI was superior to FOLFOX in upper left half. FOLFOX was superior to FOLFIRI in lower right half. Open circle indicates the patients treated without chemotherapy. Closed circle indicates the patients treated with appropriate first-line regimen. Closed triangle indicates the patients treated with inappropriate first-line regimen. FOLFOX, leucovorin and fluorouracil plus oxaliplatin; FOLFIRI, leucovorin and fluorouracil plus irinotecan.

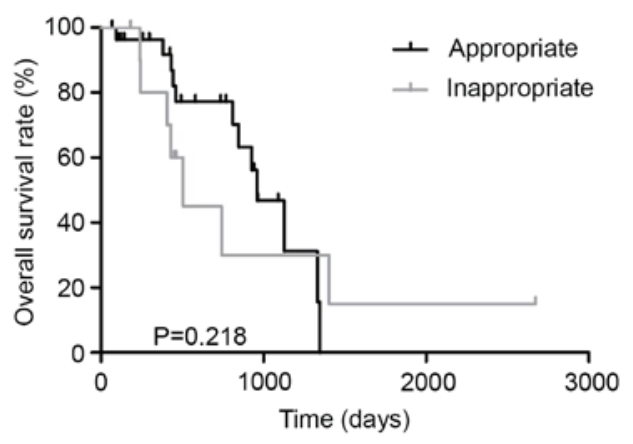

Figure 6. In all patients, overall survival rates in patients treated with appropriate first-line regimen (black line) and patients treated with inappropriate first-line regimen (gray line).

the individualized chemotherapy with molecularly-targeted anticancer agents may be implemented based on the 


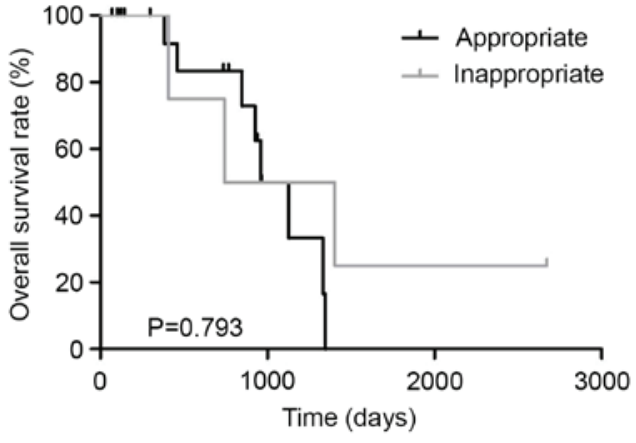

Figure 7. In dual responders, overall survival rates in patients treated with appropriate first-line regimen (black line) and patients treated with inappropriate first-line regimen (gray line).

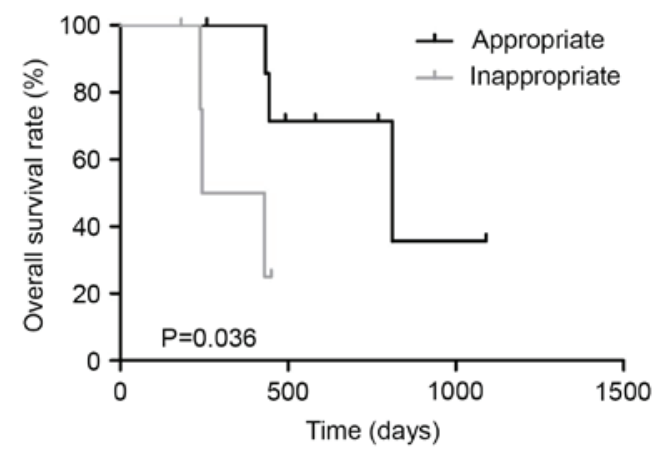

Figure 8. In poor responders, overall survival rates in patients treated with appropriate first-line regimen (black line) and patients treated with inappropriate first-line regimen (gray line).

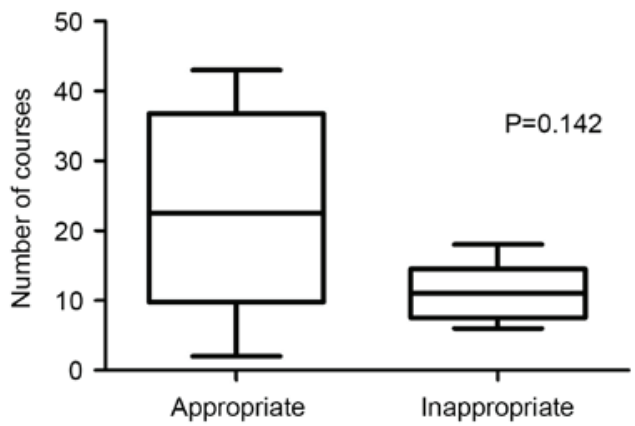

Figure 9. Frequency of chemotherapy of the patients treated with appropriate first-line regimen and the patients treated with inappropriate first-line regimen.

genetic characteristics of the individual patient. Recently, the importance of the biomarker for molecularly-targeted anticancer agents has become increasingly evident in a clinical setting (35-37). In poor responders, the biomarker for molecularly-targeted anticancer agents is required in order to improve the prognosis. Therefore, advanced studies into biomarkers are important.

There was no different prognosis between patients treated with the appropriate first-line regimen and patients treated with the inappropriate first-line regimen in dual responders. Grothey et al (38) reported that while it was not significant whether FOLFOX or FOLFIRI was administered first, it was crucial that full administration of the targeted dosages of all 3 drugs (5-FU, oxaliplatin and irinotecan) was achieved (38). However, second-line chemotherapy must be abandoned in certain patients due to disease progression, adverse effects or high medical cost in a clinical setting. The cost of molecularly-targeted anticancer agents in particular is expensive. Therefore, the number of reports on cost effectiveness analysis and cost utility analysis are rapidly increasing (10-14). In randomized controlled trials (RCTs), the rate of second-line chemotherapy enforcement in PRIME study, OPUS study, NO16966 study and FIRE-3 study was 62, $<50,53$ and $69.9 \%$, respectively (39-42). Even in those recent RCTs with strict eligible standard, second-line chemotherapy could not be carried out in $>30 \%$ of the patients. First-line chemotherapy is usually administered over a long period of time (9). In addition, the response rate of the first-line chemotherapy is typically higher compared with second-line chemotherapy $(43,44)$. Therefore, selection of a more effective regimen as the first-line chemotherapy using CD-DST is extremely important even for dual responder patients in a clinical setting. The present study has already reported the following: When the clinical response rates of FOLFOX and FOLFIRI were 50\%, responders were identified using the median based on the histograms of the individual growth IRs. The efficacies of FOLFOX and FOLFIRI were not exactly equivalent in all the individuals. By using CD-DST, it was possible to individualize the first line chemotherapy and may also improve the prognosis of patients with unresectable CRC.

In poor responders, there were significant differences of prognosis between patients treated with appropriate first-line regimen and patients treated with an inappropriate first-line regimen. Moreover, more chemotherapy in patients treated with the appropriate first-line regimen was performed. It is crucial to administer the appropriate first-line regimen. Administration of a more effective first-line regimen leads to prolonging the period of first-line chemotherapy and increases the total number of chemotherapy cycles. This indicates the importance for the detection of poor responders and the selection of first-line chemotherapy using CD-DST.

There were certain limitations to the present cohort study. Firstly, the sample size was small; a larger sample size would have improved the quality of the data. Secondly, in the present study, the periods of observation of 4 patients in dual responder were shorter ( $<150$ days) compared with other patients. The short periods of observation may influence statistical analysis. Moreover, the present study was not randomized. The individualization of first-line chemotherapy using CD-DST requires additional prospective randomized studies.

In conclusion, the results from the present study suggest that the administration of the recommended first-line regimen using CD-DST for patients with unresectable CRC is important for improvement in prognosis. It is important to administrate appropriate first-line regimen, particularly in poor responders.

\section{References}

1. Ferlay J, Soerjomataram I, Dikshit R, Eser S, Mathers C, Rebelo M, Parkin DM, Forman D and Bray F: Cancer incidence and mortality worldwide: Sources, methods and major patterns in GLOBOCAN 2012. Int J Cancer 136: E359-E386, 2015. 
2. Adam R, De Gramont A, Figueras J, Guthrie A Kokudo N, Kunstlinger F, Loyer E, Poston G, Rougier P, Rubbia-Brandt L, et al: The oncosurgery approach to managing liver metastases from colorectal cancer: A multidisciplinary international consensus. Oncologist 17: 1225-1239, 2012.

3. Jones RP, Stättner S, Sutton P, Dunne DF, McWhirter D, Fenwick SW, Malik HZ and Poston GJ: Controversies in the oncosurgical management of liver limited stage IV colorectal cancer. Surg Oncol 23: 53-60, 2014.

4. NCCN: NCCN Clinical Practice Guidelines in Oncology. Colon Cancer. Version 1. 2017. http://www.nccn.org. Accessed February 24, 2017

5. Van Cutsem E, Cervantes A, Adam R, Sobrero A, Van Krieken JH, Aderka D, Aranda Aguilar E, Bardelli A, Benson A, Bodoky G, et al: ESMO consensus guidelines for the management of patients with metastatic colorectal cancer. Ann Oncol 27: 1386-1422, 2016.

6. Fakih MG: Metastatic colorectal cancer: Current state and future directions. J Clin Oncol 33: 1809-1824, 2015.

7. Venook A, Niedzwiecki D, Lenz H, Mahoney M, Innocenti $F$ O'Neil B, Shaw J, Polite B, Hochster H, Goldberg R, et al: CALGB/SWOG 80405: Analysis of patients undergoing surgery as part of treatment strategy. Ann Oncol 25: 1-41, 2014

8. Recondo G Jr, Díaz-Cantón E, de la Vega M, Greco M, Recondo G Sr and Valsecchi ME: Advances and new perspectives in the treatment of metastatic colon cancer. World J Gastrointest Oncol 6: 211-224, 2014.

9. Tournigand C, André T, Achille E, Lledo G, Flesh M, Mery-Mignard D, Quinaux E, Couteau C, Buyse M Ganem G, et al: FOLFIRI followed by FOLFOX6 or the reverse sequence in advanced colorectal cancer: A randomized GERCOR study. J Clin Oncol 22: 229-237, 2004.

10. Leung HW, Chan AL, Leung MS and Lu CL: Systematic review and quality assessment of cost-effectiveness analysis of pharmaceutical therapies for advanced colorectal cancer. Ann Pharmacother 47: 506-518, 2013.

11. Parkin DM, Bray F, Ferlay J and Pisani P: Global cancer statistics, 2002. CA Cancer J Clin 55: 74-108, 2005.

12. Drummond MF, Sculpher MJ, Torrance GW, O'Brien BJ and Stoddart GL: Methods for the economic evaluation of health care programmes. Oxford University Press, New York, NY, 2005.

13. Manca A, Asseburg C, Bravo Vergel Y, Seymour MT, Meade A, Stephens R, Parmar M and Sculpher MJ: The cost-effectiveness of different chemotherapy strategies for patients with poor prognosis advanced colorectal cancer (MRC FOCUS). Value Health 15: 22-31, 2012.

14. Lange A, Prenzler A, Frank M, Kirstein M, Vogel A and von der Schulenburg JM: A systematic review of cost-effectiveness of monoclonal antibodies for metastatic colorectal cancer. Eur J Cancer 50: 40-49, 2014.

15. Ochiai T, Nishimura K, Watanabe T, Kitajima M, Hashiguchi T, Nakatani A, Marusasa T, Muraki A, Nagaoka I and Futagawa S: Leucovorin and fluorouracil plus oxaliplatin or leucovorin and fluorouracil plus irinotecan as individualized first-line therapy based on a drug sensitivity test. Exp Ther Med 1: 325-329, 2010.

16. Ochiai T, Nishimura K, Watanabe T, Kitajima M, Nakatani A, Inou T, Washio M, Sakuyama N, Sato T, Kishine K, et al: Individualized chemotherapy for colorectal cancer based on the collagen gel droplet-embedded drug sensitivity test. Oncol Lett 4: 621-624, 2012

17. Kobayashi H, Tanisaka K, Doi O, Kodama K, Higashiyama M, Nakagawa H, Miyake M, Taki T, Hara S, Yasutomi M, et al: An in vitro chemosensitivity test for solid tumors using collagen gel droplet embedded cultures. Int J Oncol 11: 449-455, 1997.

18. Kobayashi H, Higashiyama M, Minamigawa K, Tanisaka K, Takano T, Yokouchi H, Kodama K and Hata T: Examination of in vitro chemosensitivity test using collagen droplet culture method with colorimetric endpoint quantification. Jpn J Cancer Res 92: 203-210, 2001.

19. de Gramont A, Figer A, Seymour M, Homerin M, Hmissi A, Cassidy J, Boni C, Cortes-Funes H, Cervantes A, Freyer G, et al: Leucovorin and fluorouracil with or without oxaliplatin as first-line treatment in advanced colorectal cancer. J Clin Oncol 18: 2938-2947, 2000

20. Goldberg RM, Sargent DJ, Morton RF, Fuchs CS Ramanathan RK, Williamson SK, Findlay BP, Pitot HC and Alberts SR: A randomized controlled trial of fluorouracil plus leucovorin, irinotecan and oxaliplatin combinations in patients with previously untreated metastatic colorectal cancer. J Clin Oncol 22: 23-30, 2004.
21. Douillard JY, Cunningham D, Roth AD, Navarro M, James RD, Karasek P, Jandik P, Iveson T, Carmichael J, Alakl M, et al: Irinotecan combined with fluorouracil compared with fluorouracil alone as first-line treatment for metastatic colorectal cancer: A multicentre randomized trial. Lancet 355: 1041-1047, 2000.

22. Ochiai T, Nishimura K, Noguchi H, Kitajima M, Tsukada A, Watanabe E, Nagaoka I and Futagawa S: Prognostic impact of orotate phosphoribosyl transferase among 5-fluorouracil metabolic enzymes in resectable colorectal cancers treated by oral 5-fluorouracil-based adjuvant chemotherapy. Int J Cancer 118: 3084-3088, 2006.

23. Aki F, Bando Y, Takahashi T, Uehara H, Numoto S, Ito S, Sasa M and Izumi K: A retrospective study on TS mRNA expression and prediction of the effects of adjuvant oral 5-fluorouracil in breast cancer. Oncol Lett 1: 981-987, 2010.

24. Komori S, Osada S, Tomita H, Nishio K, Kumazawa I, Tachibana S, Tsuchiya J and Yoshida K: Predictive value of orotate phosphoribosyltransferase in colorectal cancer patients receiving 5-FU-based chemotherapy. Mol Clin Oncol 1: 453-460, 2013.

25. Ochiai T, Umeki M, Miyake H, Iida T, Okumura M, Ohno K, Sakamoto M, Miyoshi N, Takahashi M, Tsumura H, et al: Impact of 5-fluorouracil metabolizing enzymes on chemotherapy in patients with resectable colorectal cancer. Oncol Rep 32: 887-892, 2014

26. Sasako M, Terashima M, Ichikawa W, Ochiai A, Kitada K, Kurahashi I, Sakuramoto S, Katai H, Sano T and Imamura H: Impact of the expression of thymidylate synthase and dihydropyrimidine dehydrogenase genes on survival in stage II/III gastric cancer. Gastric Cancer 18: 538-548, 2015.

27. Kawakami H, Zaanan A and Sinicrope FA: Implications of mismatch repair-deficient status on management of early stage colorectal cancer. J Gastrointest Oncol 6: 676-684, 2015.

28. Jung SH, Kim SH and Kim JH: Prognostic impact of microsatellite instability in colorectal cancer presenting with mucinous, signet-ring, and poorly differentiated cells. Ann Coloproctol 32: 58-65, 2016.

29. Ashktorab H, Ahuja S, Kannan L, Llor X, Ellis NA, Xicola RM, Laiyemo AO, Carethers JM, Brim $\mathrm{H}$ and Nouraie M: A meta-analysis of MSI frequency and race in colorectal cancer. Oncotarget 23: 34546-34557, 2016.

30. van Kuilenburg AB and Maring JG: Evaluation of 5-fluorouracil pharmacokinetic models and therapeutic drug monitoring in cancer patients. Pharmacogenomics 14: 799-811, 2013

31. Paci A, Veal G, Bardin C, Levêque D, Widmer N, Beijnen J, Astier A and Chatelut E: Review of therapeutic drug monitoring ofanticancer drugs part 1-Cytotoxics. Eur J Cancer 50: 2010-2019, 2014

32. Derissen EJ, Jacobs BA, Huitema AD, Rosing H, Schellens JH and Beijnen JH: Exploring the intracellular pharmacokinetics of the 5-fluorouracil nucleotides during capecitabine treatment. $\mathrm{Br}$ J Clin Pharmacol 81: 949-957, 2016.

33. Yang R, Zhang Y, Zhou H, Zhang P, Yang P, Tong Q, Lyu Y and Han Y: Individual 5-fluorouracil dose adjustment via pharmacokinetic monitoring versus conventional body-area-surface method: A meta-analysis. Ther Drug Monit 38: 79-86, 2016.

34. Lee JJ, Beumer JH and Chu E: Therapeutic drug monitoring of 5-fluorouracil. Cancer Chemother Pharmacol 78: 447-464, 2016.

35. Bencsikova B, Bortlicek Z, Halamkova J, Ostrizkova L, Kiss I, Melichar B, Pavlik T, Dusek L, Valik D, Vyzula R and Zdrazilova-Dubska L: Efficacy of bevacizumab and chemotherapy in the first-line treatment of metastatic colorectal cancer: Broadening KRAS-focused clinical view. BMC Gastroenterol 15: 37, 2015.

36. Hecht JR, Cohn A, Dakhil S, Saleh M, Piperdi B, Cline-Burkhardt $M$, Tian Y and Go WY: SPIRITT: A randomized, multicenter, phase II study of panitumumab with FOLFIRI and bevacizumab with FOLFIRI as second-line treatment in patients with unresectable wild type KRAS metastatic colorectal cancer. Clin Colorectal Cancer 14: 72-80, 2015.

37. Sartore-Bianchi A, Trusolino L, Martino C, Bencardino K, Lonardi S, Bergamo F, Zagonel V, Leone F, Depetris I, Martinelli E, et al: Dual-targeted therapy with trastuzumab and lapatinib in treatment-refractory, KRAS codon 12/13 wild-type, HER2-positive metastatic colorectal cancer (HER ACLES): A proof-of-concept, multicentre, open-label, phase 2 trial. Lancet Oncol 17: 738-746, 2016. 
38. Grothey A, Sargent D, Goldberg RM and Schmoll HJ: Survival of patients with advanced colorectal cancer improves with the availability of fluorouracil-leucovorin, irinotecan and oxaliplatin in the course of treatment. J Clin Oncol 22: 1209-1214, 2004.

39. Cassidy J, Clarke S, Díaz-Rubio E, Scheithauer W, Figer A, Wong R, Koski S, Lichinitser M, Yang TS, Rivera F, et al: Randomized phase III study of capecitabine plus oxaliplatin compared with fluorouracil/folinic acid plus oxaliplatin as first-line therapy for metastatic colorectal cancer. J Clin Oncol 26: 2006-2012, 2008.

40. Douillard JY, Siena S, Cassidy J, Tabernero J, Burkes R, Barugel M, Humblet Y, Bodoky G, Cunningham D, Jassem J, et al: Randomized, phase III trial of panitumumab with infusional fluorouracil, leucovorin and oxaliplatin (FOLFOX4) versus FOLFOX4 alone as first-line treatment in patients with previously untreated metastatic colorectal cancer: The PRIME study. J Clin Oncol 28: 4697-4705, 2010.

41. Bokemeyer C, Bondarenko I, Hartmann, de Braud F, Schuch G, Zubel A, Celik I, Schlichting M and Koralewski P: Efficacy according to biomarker status of cetuximab plus FOLFOX-4 as first-line treatment for metastatic colorectal cancer: The OPUS study. An Oncol 22: 1535-1546, 2011.
42. Modest DP, Stintzing S, von Weikersthal LF, Decker T, Kiani A, Vehling-Kaiser U, Al-Batran SE, Heintges T, Lerchenmüller C, Kahl C, et al: Impact of subsequent therapies on outcome of the FIRE-3/AIO KRK0306 trial: First-line therapy with FOLFIRI plus cetuximab or bevacizumab in patients with KRAS wild-type tumors in metastatic colorectal cancer. J Clin Oncol 33: 3718-3726, 2015.

43. Haller DG, Rothenberg ML, Wong AO, Koralewski PM, Miller WH Jr, Bodoky G, Habboubi N, Garay C and Olivatto LO: Oxaliplatin plus irinotecan compared with irinotecan alone as second-line treatment after single-agent fluoropyrimidine therapy for metastatic colorectal carcinoma. J Clin Oncol 26: 4544-4550, 2008.

44. Koopman M, Antonini NF, Douma J, Wals J, Honkoop AH, Erdkamp FL, de Jong RS, Rodenburg CJ, Vreugdenhil G, Loosveld OJ, et al: Sequential versus combination chemotherapy with capecitabine, irinotecan, and oxaliplatin in advanced colorectal cancer (CAIRO): A phase III randomised controlled trial. Lancet 370: 135-142, 2007. 